\title{
Sistema de Comunicação Alternativa para Letramento de Pessoas com Autismo - SCALA Tablet
}

\author{
Roberto Franciscatto ${ }^{1}$, Maria Rosangela Bez ${ }^{1}$, Liliana Maria Passerino ${ }^{1}$ \\ ${ }^{1}$ CINTED (Centro Interdisciplinar de Novas Tecnologias na Educação) - Universidade \\ Federal do Rio Grande do Sul (UFRGS) - 90040-060 - Porto Alegre, RS - Brasil \\ \{roberto.franciscatto, bezrosangela, lpasserino\}@gmail.com
}

\begin{abstract}
Resumo. O trabalho em questão objetiva descrever sobre o desenvolvimento de um Sistema de Comunicação Alternativa para Letramento de Pessoas com Autismo, denominado SCALA Tablet e seu contexto de uso enquanto Tecnologia Assistiva para apoio ao processo de desenvolvimento da comunicação e do letramento do aluno com TEA (Transtorno do Espectro Autista). Apresenta-se no decorrer do texto o cenário de uso do aplicativo, descreve-se o processo de desenvolvimento de software, os principais recursos disponiveis no aplicativo, bem como, as características do protótipo em questão. Por fim, são expostas as contribuições pedagógicas do protótipo, síntese dos resultados obtidos e os trabalhos futuros.
\end{abstract}

\section{Cenário de Uso}

A busca de técnicas, recursos e estratégias para a autonomia de pessoas com deficiência passou a se intensificar a partir da Lei de inclusão (BRASIL, 2008). Diante de tal cenário, uma gama de recursos de software, como sistemas (web, desktop e mobile) foi desenvolvida, como forma de oferecer serviços que pudessem auxiliar pessoas com diferentes tipos de necessidades especiais. Aliado a revolução tecnológica da atualidade, os recursos para este público-alvo se efetivam através das tecnologias assistivas. No caso deste protótipo desenvolvido, as de comunicação alternativa, em especial ao TEA (Transtorno do Espectro Autista) através do software SCALA Tablet Sistema de Comunicação Alternativa para Letramento de pessoas com Autismo, composto por recursos tecnológicos mais uma metodologia de uso.

\subsection{Tecnologias Assistivas de Comunicação Alternativa}

A Comunicação Alternativa (CA) é uma das áreas da Tecnologia Assistiva, que apoia o desenvolvimento de uma comunicação mais autônoma das pessoas com déficits nesse âmbito. A CA preocupa-se com técnicas, processos e ferramentas que auxiliem a comunicação, como apoio, complementação ou substituição da fala. Seu uso justifica-se não pelo suporte midiático adotado, mas pelas estratégias e técnicas comunicativas que podem promover a autonomia dos sujeitos em situações de comunicação (TETZCHNER e MARTINSEN, 2000, WALTER, 2011).

No caso do autismo, os déficits de comunicação podem se manifestar com alterações no uso, na forma ou no conteúdo da linguagem na pragmática e em menor medida, no nível sintático, morfossintático, fonológico ou fonético. Desse modo, a 


\section{CBIE-LACLO 2015}

Anais dos Workshops do IV Congresso Brasileiro de Informática na Educação (CBIE 2015)

importância de utilizar um sistema de CA está focada mais em processos de compreensão e de produção de sentidos do que em produção sonora ou morfossintática (PASSERINO, 2008).

Estudos recentes desenvolvidos por Bez e Passerino (2009), Bez (2010, 2014), Ávila e Passerino (2011b) e Ávila (2011), envolvendo o uso de CA com sujeitos com Transtorno do Espectro Autista, apresentam resultados importantes. Esses resultados são significativos, especialmente, quando se apoia os processos de CA no uso de tecnologias digitais para o desenvolvimento da comunicação e da interação social de sujeitos com autismo.

Softwares de CA têm sido desenvolvidos para desktops, como por exemplo, Boardmaker, Plaphoons, E-triloquist, Amplisoft, dentre outros, ou para uso na web, de forma online como o Askability, Symbolworld, PICTO4ME, para uso direto com o usuário ou utilizados na elaboração de atividades ou pranchas de comunicação. Assim como, para uso em dispositivos móveis, como por exemplo, AraBoard, Grid Player, My Voice My Words, PictoDriod Life, Dílo, dentre outros.

Observa-se, entretanto, que a grande maioria dos aplicativos para dispositivos móveis ou são pagos ou não foram desenvolvidos pensando-se no público do TEA (Transtorno do Espectro Autista) que estejam em fase inicial de letramento (propósito do SCALA Tablet). Dentro desta perspectiva surge o SCALA Tablet, que objetiva apoiar no desenvolvimento da comunicação, interação social, inclusão e letramento de sujeitos com TEA. Numa perspectiva de professores atuantes como mediadores de práticas educativas com esses sujeitos e na participação intensa da família para utilização e adaptação dessas estratégias e recursos.

\section{Desenvolvimento}

O protótipo SCALA Tablet foi desenvolvido como um aplicativo para tablets baseados no sistema operacional Android (disponível a partir da versão 3.0 deste sistema operacional), originário do sistema SCALA Web (scala.ufrgs.br). A opção de escolha pelo desenvolvimento para dispositivos baseados no sistema operacional Android se dá pela ampla utilização deste sistema no contexto dos dispositivos móveis. O protótipo SCALA Tablet, foi desenvolvido para dispositivos de 07 (sete) polegadas ou mais, com resolução de vídeo de $600 \times 1024$ pixels. A versão deste protótipo móvel foi desenvolvida, seguindo os padrões de projeto (Design Pattern), conforme recomendação da equipe de Desenvolvimento Google, desenvolvedora da plataforma Android SDK. Entre os principais padrões utilizados, destacam-se: Model-ViewController (MVC); internacionalização; utilização de APIs padronizadas (text-to-Sprech (TTS); compartilhamento de recursos para exportação de imagens; Web Services REST; interpretação e geração de arquivos (XML).

Quanto à validação do protótipo o mesmo foi testado primeiramente através do simulador nativo da linguagem Android, denominado Android Virtual Device (AVD) Manager, onde foi possível simular diferentes tipos de dispositivos, bem como, seus atributos (nível da API suportada, tipo de dispositivo, tamanho da tela, tamanho do espaço interno de armazenamento, entre outros). 


\section{CBIE-LACLO 2015}

Anais dos Workshops do IV Congresso Brasileiro de Informática na Educação (CBIE 2015)

Além dos simuladores, três diferentes tablets foram utilizados das marcas Samsung, Asus e Motorola, como forma de validar o aplicativo e identificar possíveis problemas. Foi realizada a proposta de um contexto controlado (experimental, acompanhado por duas pedagogas em ambiente escolar) como forma de investigar a interação entre pares, com a inserção dos três sujeitos em um mesmo espaço social (crianças com transtorno do espectro autista, com idade entre 03 e 05 anos) e sua experimentação junto ao protótipo desenvolvido. Os encontros nesse contexto ocorreram nas sextas-feiras de manhã, durante um período de 06 meses, totalizando 38 intervenções.

\section{Apresentação do software}

Conforme descrito na seção anterior o SCALA Tablet é uma derivação do sistema SCALA web (disponível em scala.ufrgs.br), desenvolvimento para o sistema operacional Android e destina-se a alfabetização de crianças com TEA. A interface do software foi desenvolvida em paralelo com a análise de requisitos e modelagem. Nesse sentido, Preece, Rogers \& Shap (2007) e Passerino, Bez (2013) contribuíram para a organização de um design focado no usuário em relação aos outros (pessoas) e em contextos de uso, tendo este como ponto fundamental em cada decisão tomada pelo grupo. Esta metodologia de desenvolvimento foi intitulada de Design Centrado em Contextos de Uso - DCC, maiores informações a respeito podem ser encontradas em Bez (2014). O protótipo em questão apresenta dois recursos principais: o módulo prancha de comunicação e o módulo narrativas visuais, que serão descritos na sequência.

\subsection{Módulo prancha - SCALA Tablet}

O módulo prancha na versão dispositivo móvel destina-se a construção de atividades variadas elaboradas por professores, familiares e profissionais que atuam com o sujeito com deficiência ou até mesmo pelo próprio sujeito com déficit. O diagrama de casos de uso apresenta as funcionalidades esperadas para este módulo e seus requisitos funcionais e não funcionais, descritas na figura 1 .

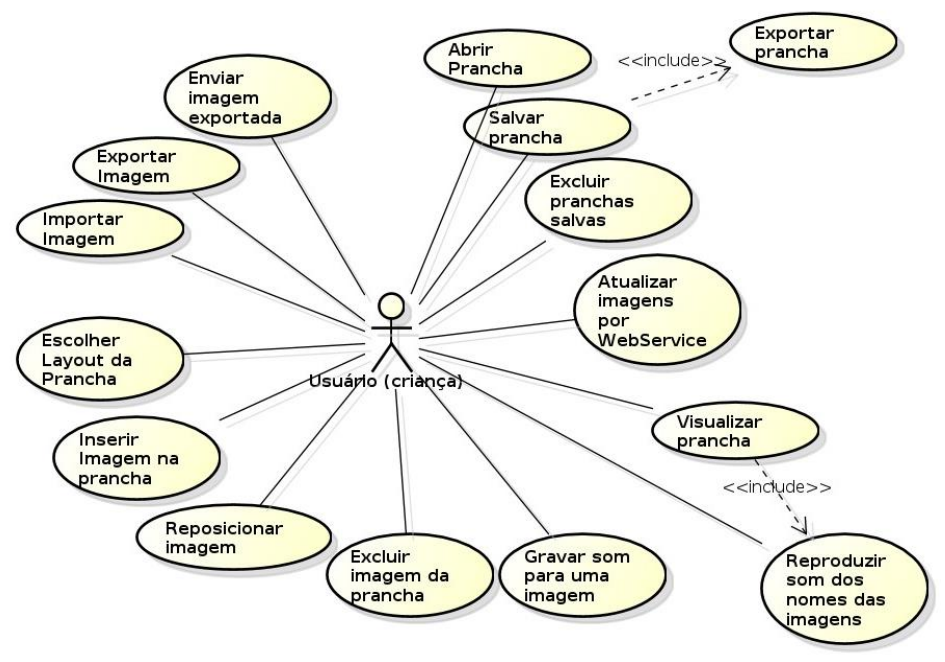

Figura 1: Casos de uso SCALA dispositivo móvel - prancha 
O ator principal do sistema é a criança ou o adulto que irá manipular o Tablet. Ele pode realizar todos os casos de uso previstos pelo sistema. Com referência ao uso do software, o diagrama de atividades a seguir apresenta o processo padrão de utilização do sistema. Ele pode sofrer mudanças, de acordo com o interesse do usuário (figura 2).

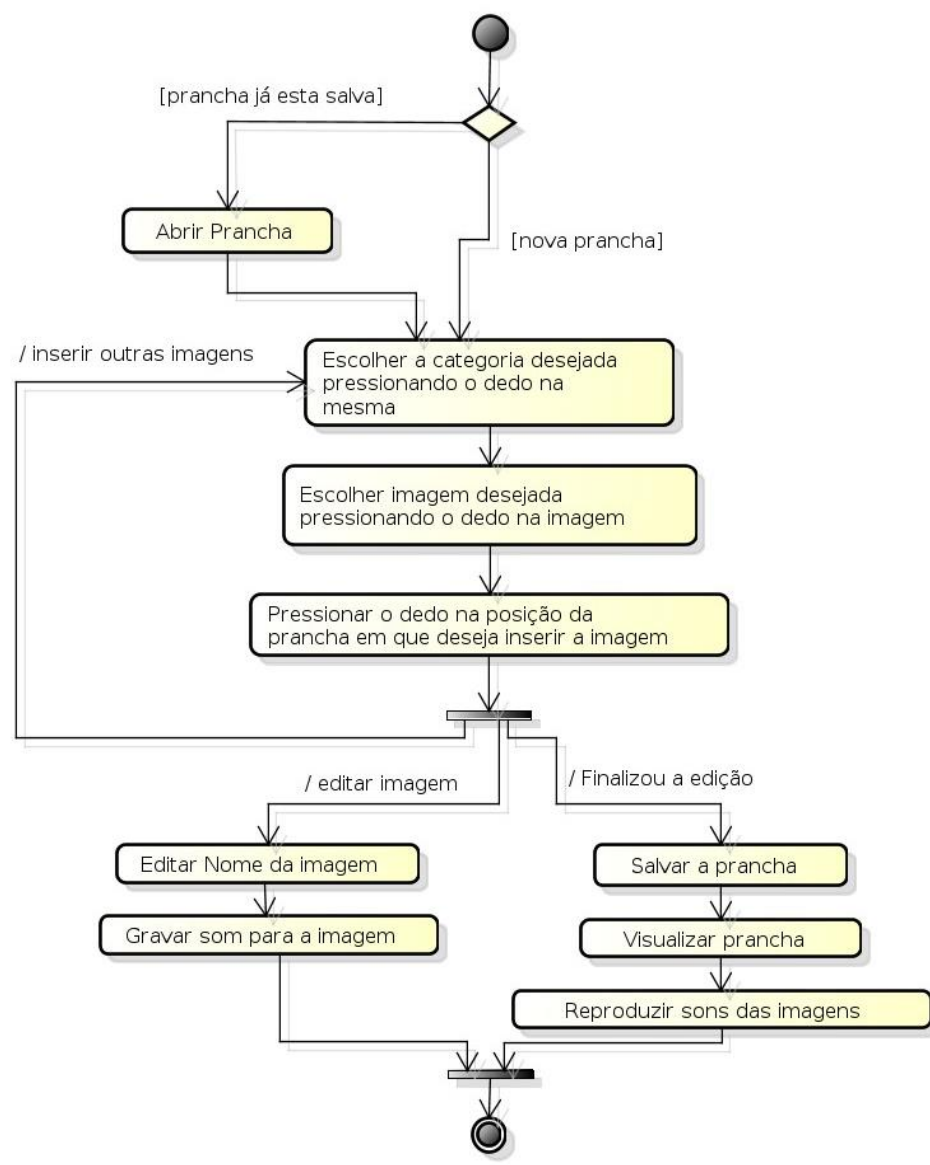

Figura 2: Diagrama de atividades dispositivo móvel - prancha

Na figura 3 tem-se a representação visual do módulo prancha para dispositivo móvel.

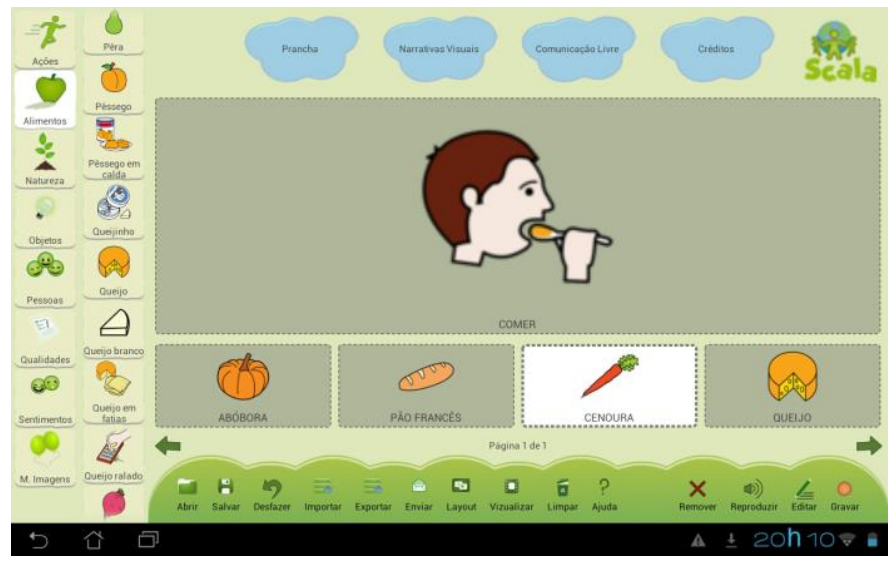

Figura 3: Representação visual dispositivo móvel - prancha

O módulo prancha para dispositivo móvel (SCALA Tablet) possui os seguintes recursos: menu de categorias (divididas em ações, alimentos, natureza, objetos, pessoas, 
qualidades, sentimentos e minhas imagens, totalizando 08 categorias), onde é possível localizar um pictograma para ser "arrastado" até a prancha central; menu de opções da prancha (abrir, salvar, desfazer, importar, exportar, enviar, layout, visualizar, limpar e ajuda) onde é possível manipular a prancha e indicar ação que será realizada sobre a mesma; menu superior (prancha, narrativas visuais, comunicação livre e créditos) onde tem-se acesso aos recursos principais do protótipo SCALA Tablet; e por fim no centro da figura 3, a prancha de comunicação onde são construídas as pranchas propriamente ditas.

Um recurso interessante na versão SCALA Tablet para o sistema SCALA web é a substituição da funcionalidade "imprimir" pela funcionalidade "enviar". Essa alteração ocorreu devido à dificuldade de impressão diretamente do dispositivo móvel. Dessa forma, pelo "enviar", é possível encaminhar a prancha produzida via bluetooth, e-mail, mensagens, rede sociais, entre outros.

\subsection{Módulo narrativas visuais - SCALA Tablet}

O módulo narrativas visuais do SCALA Tablet permite ao utilizador a construção de histórias colaborativas e personalizadas, conforme as necessidades do usuário. Este módulo diferencia-se do anterior no sentido de permitir mais opções personalizadas ao usuário, como por exemplo, a inserção de títulos às histórias que serão criadas, menu de balões (formatos circular, quadrado e nuvem) para inserção de textos (refletindo conversas entre os agentes presentes na história a ser criada), diferentes tipos de cenários que podem ser utilizados, bem como, reprodução de áudio dos diálogos criados.

O diagrama de casos de uso baseia-se nos requisitos e apresenta as funcionalidades e características funcionais do módulo narrativas visuais, sendo as mesmas descritas na figura 4.

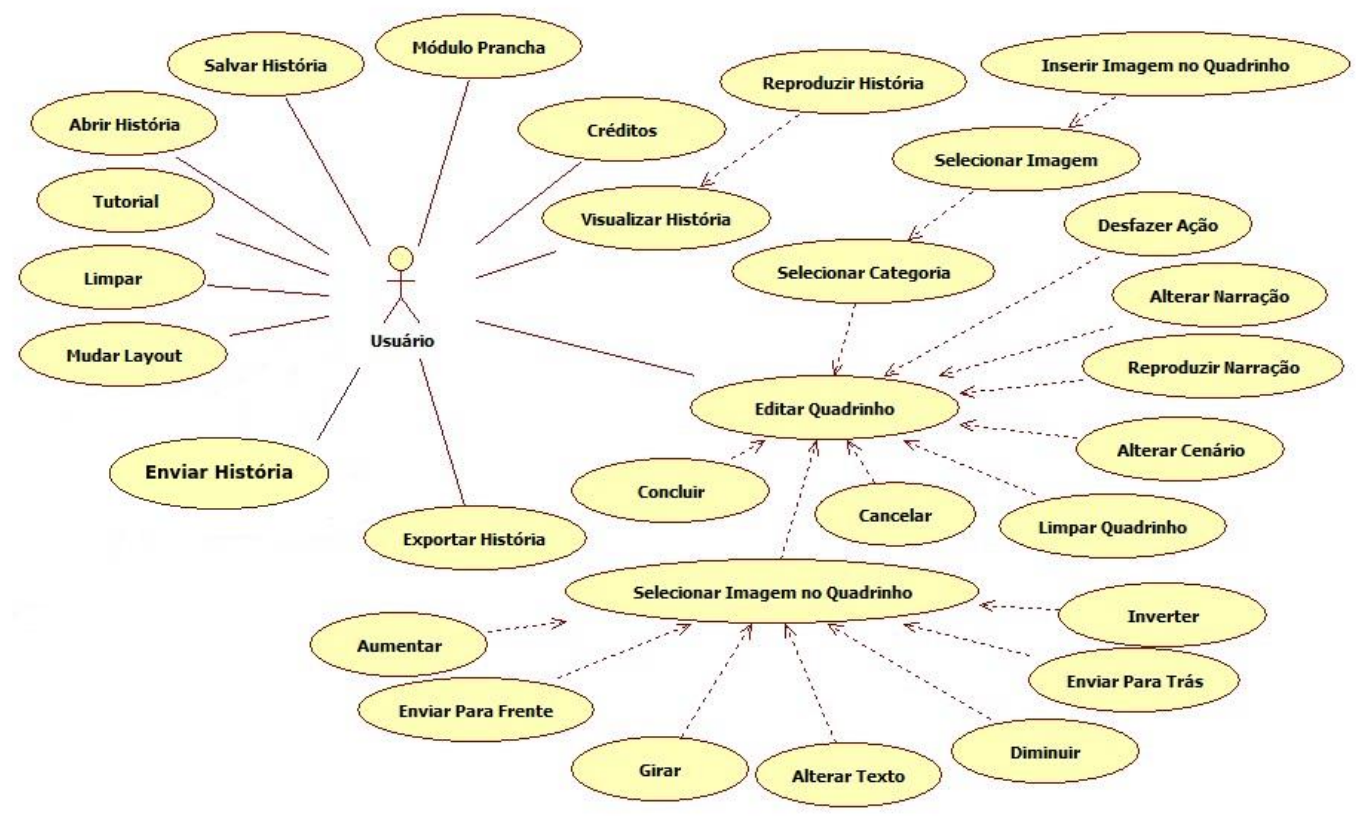

Figura 4: Casos de uso - módulo narrativas visuais 


\section{CBIE-LACLO 2015}

Anais dos Workshops do IV Congresso Brasileiro de Informática na Educação (CBIE 2015)

O ator principal do sistema é a criança ou o adulto que irá manipular o Tablet, no SCALA. Ele pode realizar todos os casos de uso previstos pelas funcionalidades do sistema. O módulo narrativas visuais para o protótipo SCALA Tablet pode ser visualizado na figura 5 .
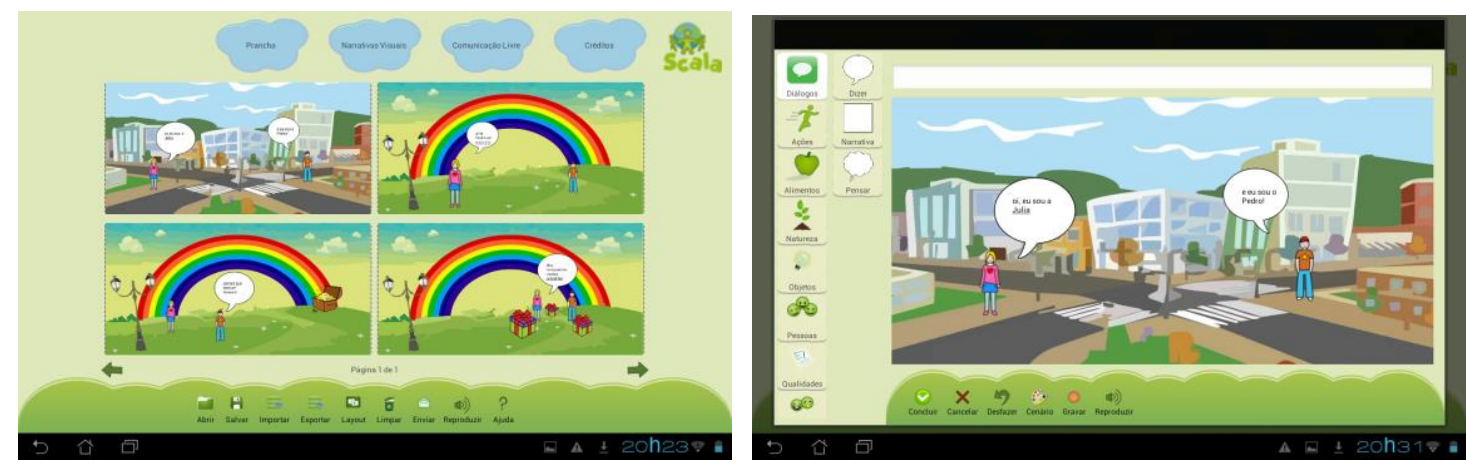

Figura 5: Layout e modo de edição do módulo narrativas visuais - SCALA Tablet

O SCALA Tablet pode ser encontrado no site do projeto SCALA (http://scala.ufrgs.br/siteScala/projeto/?q=node/52) onde é possível ter acesso a página do aplicativo, informações técnicas, configurações, download e processo de instalação do mesmo. Os tutoriais passo a passo podem ser encontrados no próprio software, tanto no módulo Prancha, como no módulo Narrativas Visuais, no menu inferior das funcionalidades no item Ajuda. O protótipo SCALA Tablet é livre, com licença Creative Commons, atribuição não comercial e tem como base os pictogramas do ARASSAC ${ }^{1}$, desenvolvido por Cidmantas Zemleris.

\section{Considerações finais}

As principais contribuições pedagógicas acerca do protótipo SCALA Tablet, foram a construção de um sistema de comunicação alternativa, para o ambiente mobile, que além de uma tecnologia assistiva em si, necessitou-se de uma forma de desenvolvimento própria, que contemplasse crianças com autismo, em faixa etária da educação infantil, com adequação há variados contextos que elas estivessem inseridas. Entretanto, como se desejava uma base sócio histórica, foi essencial o foco também dessa criança em relação às outras pessoas que participavam desses contextos.

Ainda, no processo do desenvolvimento do sistema, foi indispensável a atuação nos três contextos escolhidos (familiar, escolar e experimental), envolvendo crianças com autismo através de estratégias de uso do sistema. Para tal, foi proposta uma metodologia de Ações Mediadoras que mostrava, não uma "receita de bolo" mas, etapas que poderiam ser seguidas para apoiar, tanto o conhecimento dos sujeitos e dos contextos, como a escolha de recursos a serem utilizados, através de ações (atividades).

Considerando a pesquisa realizada em paralelo ao desenvolvimento do protótipo final, a mesma focou na resolução de um problema, no caso, o desenvolvimento de um sistema de comunicação alternativa para atender uma carência do mercado de

\footnotetext{
${ }^{1}$ Portal Aragonés de la Comunicación Aumentativa y Alternativa - ARASAAC (http://arasaac.org).
} 


\section{CBIE-LACLO 2015}

Anais dos Workshops do IV Congresso Brasileiro de Informática na Educação (CBIE 2015)

tecnologias assistivas gratuitas brasileiras ou em língua portuguesa, para apoio de crianças com autismo. Da mesma forma, foram colocadas em prática ações mediadoras não triviais, utilizadas em diversos contextos dos sujeitos foco.

Como resultados da validação do software, no que se refere à análise da avaliação pedagógica, os avaliadores consideraram que o sistema SCALA Tablet tem potencial para ser utilizado no processo de alfabetização/letramento e em diferentes atividades elaboradas para apoio à oralidade. A sua proposta educacional é clara, considerando que é adequado para pessoas que não sabem ler, e que, possui imagens que atendam à construção de pranchas de comunicação alternativa e histórias, capazes de efetivar o desenvolvimento da comunicação, interação e inclusão do aluno, através do desenvolvimento de atividades colaborativas.

Os resultados da análise do desenvolvimento do sistema SCALA Tablet mostraram que a metodologia que foi utilizada para sua elaboração foi eficiente. Isso para o intuito a que se propôs, de apoiar processos de inclusão de sujeitos com autismo na faixa etária de 3-5 anos, com possibilidade de uso colaborativo, e de apoio ao processo de alfabetização/letramento e da comunicação dos usuários. Dessa forma, crêse ter atendido ao objetivo de análise do sistema SCALA Tablet.

Com os resultados obtidos nas avaliações do SCALA Tablet, há diversas sugestões de melhorias e ampliações propostas pelos professores, como, por exemplo, um sistema de busca que facilite e agilize a procura de imagens. Ainda deve-se pensar em uma forma de integração do banco de imagens do sistema SCALA web com o protótipo SCALA Tablet. Ainda, como projetos futuros pretende-se estender a aplicação para o conceito webapp e disponibilizar o mesmo para outras versões de sistemas operacionais de dispositivos móveis, como por exemplo, iOS (Apple) e Windows Phone (Microsoft).

\section{Referências}

AVILA, B. G. Comunicação aumentativa e alternativa para o desenvolvimento da oralidade de pessoas com autismo. Dissertação. Faculdade de Educação. Programa de Pós-Graduação em Educação. UFRGS. Porto Alegre: 2011.

AVILA, B. G; PASSERINO, L. M. Comunicação Aumentativa e Alternativa e Autismo: desenvolvendo estratégias por meio do SCALA. In: Anais VI Seminário Nacional de Pesquisa em Educação especial: Práticas Pedagógicas na educação Especial: multiplicidade do atendimento educacional especializado, v. 1. p. 1-10. $2011 \mathrm{~b}$.

BEZ, M. R.; Passerino, L. M. Applying Alternative and Augmentative Communication to an inclusive group. In: WCCE 2009 - Education and Technology for a Better World Monday, 2009, Bento Gonçalves. WCCE 2009 Proceedings - Education and Technology for a Better World Monday. Germany: IFIP WCCE. v. 1. p. 164-174. 2009.

BEZ, M. R. Comunicação Aumentativa e Alternativa para sujeitos com Transtornos Globais do Desenvolvimento na promoção da expressão e intencionalidade por meio de Ações Mediadoras. Dissertação. Programa de Pós-Graduação em Educação - 
Faculdade de Educação. Universidade Federal Do Rio Grande Do Sul. Porto Alegre, 2010 .

BEZ, M. R. (2014) Sistema de comunicação alternativa para processos de inclusão em autismo: uma proposta integrada de desenvolvimento em contextos para aplicações móveis e web. 286 f. Tese (Doutorado em Informática na Educação) - Programa de Pós-Graduação em Informática na Educação, Centro Interdisciplinar de Novas Tecnologias na Educação, Universidade Federal do Rio Grande do Sul, Porto Alegre.

BRASIL. Política Nacional de Educação Especial na Perspectiva da Educação Inclusiva. Brasília. MEC/Secretaria de Educação Especial 2008. Disponível em: portal.mec.gov.br/arquivos/pdf/politicaeducespecial.pdf. Acesso em: maio de 2015.

PASSERINO, Liliana Maria; SANTAROSA, Lucila M Costi. Autism and Digital Learning Environments: processes of interaction and mediation. Computers and Education. v. 51, p. 385-402, 2008.

PASSERINO, Liliana Maria; BEZ, Maria Rosangela. Building an Alternative Communication System for literacy of children with autism (SCALA) with ContextCentered Design of Usage. In: Autism / Book 1. 2013, v. 1, p. 655-679. http://dx.doi.org/10.5772/54547.

PREECE, J.; ROGERS, Y.; SHARP, H. Design de interação: além da interação homemcomputador. Trad. Viviane Passamai. Porto Alegre: Bookmann, 2007.

TETZCHNER, S.; MARTINSEN, H. Introdução à Comunicação Aumentativa e Alternativa. Portugal: Porto, 2000.

WALTER, C. O PECS adaptado no ensino regular: uma opção de comunicação alternativa para alunos com autismo. In: Nunes, L. Quiterio, P; Walter, C.; Schimer, C.;Braun, P.(Org.) Comunicar é preciso: em busca das melhores práticas na educação do aluno com deficiência. Marilia: ABPEE, 2011 [192 p.] (p. 127-140). 